\title{
Screening and plasmons in pure and disordered single- and bilayer black phosphorus
}

\author{
Fengping Jin, ${ }^{1}$ Rafael Roldán, ${ }^{2, *}$ Mikhail I. Katsnelson, ${ }^{3}$ and Shengjun Yuan ${ }^{3, \dagger}$ \\ ${ }^{1}$ Institute for Advanced Simulation, Jülich Supercomputing Centre, Forschungszentrum Jülich, D-52425 Jülich, Germany \\ ${ }^{2}$ Instituto Madrileño de Estudios Avanzados en Nanociencia (IMDEA-Nanociencia), E-28049 Madrid, Spain \\ ${ }^{3}$ Radboud University, Institute for Molecules and Materials, Heijendaalseweg 135, NL-6525 AJ Nijmegen, The Netherlands
}

(Received 27 July 2015; revised manuscript received 7 September 2015; published 24 September 2015)

\begin{abstract}
We study collective plasmon excitations and screening of pure and disordered single- and bilayer black phosphorus (BP) beyond the low energy continuum approximation. The dynamical polarizability of phosphorene is computed using a tight-binding model that properly accounts for the band structure in a wide energy range. Electron-electron interaction is considered within the random phase approximation. Damping of the plasmon modes due to different kinds of disorder, such as resonant scatterers and long-range disorder potentials, is analyzed. We further show that an electric field applied perpendicular to bilayer phosphorene can be used to tune the dispersion of the plasmon modes. For sufficiently large electric field, the bilayer BP enters in a topological phase with a characteristic plasmon spectrum, which is gaped in the armchair direction.
\end{abstract}

DOI: 10.1103/PhysRevB.92.115440

PACS number(s): 73.21.-b, 73.22.Lp, 73.61.-r, 74.78.Fk

\section{INTRODUCTION}

Phosphorene is a new kind of two-dimensional (2D) material that can be obtained by the mechanical exfoliation method from black phosphorus (BP) films [1-6]. It is a semiconductor with direct band gap and highly anisotropic electronic and optical properties [3,7-18]. The band gap of thin BP films varies, depending on the thickness, from $0.3 \mathrm{eV}$ to around $2 \mathrm{eV}$. Furthermore, the electronic band structure of this material is very sensitive to strain [2,12,19-21], making it a promising candidate for electromechanical applications. The plasmons and screening in pristine single-layer and multilayer phosphorene were studied theoretically in Ref. [22] within a low energy $\mathbf{k} \cdot \mathbf{p}$ model, where it was found that the dispersion of the collective modes in this material strongly depends on the direction of propagation. A similar approximation was later used in Ref. [23] to study a generic double-layer anisotropic system with large interlayer distance. Collective excitations of $\mathrm{BP}$ in the presence of a quantizing magnetic field have been studied recently, finding that the excitation spectrum is discretized into a series of anisotropic magneto-excitons [24].

A fundamental issue that needs to be addressed is the effect of disorder on the electronic and optical properties of this material. Phosphorene and its layered structures are highly sensitive to the environment [5,25-27], due to its high reactivity when the samples are exposed to air. In this paper, we study the plasmon modes of disordered singleand bilayer BP by using a tight-binding (TB) model which properly accounts for the electronic band dispersion in a wide energy window [10]. Damping of plasmons due to different kinds of disorder, such as point defects (resonant scatterers) and long-range disorder potential, is analyzed. For this aim, the polarization function is calculated with the tight-binding propagation method (TBPM) [28-30], which is extremely efficient in large-scale calculations of systems with more than millions of atoms. The dielectric function is obtained within the random phase approximation (RPA). For the case of bilayer BP,

\footnotetext{
*rafael.roldan.toro@gmail.com

†s.yuan@science.ru.nl
}

we study the effect of an electric field applied perpendicular to the layers, which can be used to tune the dispersion of the plasmon modes. Finally we discuss the excitation spectrum of bilayer BP in the topological phase driven by the application of bias, in which the band structure is gapped in the armchair direction and dispersive in the zigzag direction.

The paper is organized as follows. In Sec. II we describe the method used in our calculations. The dielectric screening properties are discussed in Sec. III and the plasmon excitation spectrum of disordered BP is analyzed in Sec. IV. Our main conclusions are summarized in Sec. V.

\section{MODEL AND METHOD}

BP is a single-elemental layered crystalline material consisting of only phosphorus atoms arranged in a puckered orthorhombic lattice. As sketched in Fig. 1, single-layer phosphorene contains two atomic layers and two kinds of bonds with $2.22-\AA$ and $2.24-\AA$ bond lengths for in-plane and inter-plane P-P connections, respectively [7]. The electronic band structure around the gap can be described with a TB Hamiltonian for pristine BP with the form [10],

$$
\mathcal{H}=\sum_{i} \epsilon_{i} n_{i}+\sum_{i \neq j} t_{i j} c_{i}^{\dagger} c_{j}+\sum_{i \neq j} t_{p, i j} c_{i}^{\dagger} c_{j},
$$

where we consider five intralayer $\left(t_{1}=-1.220 \mathrm{eV}, t_{2}=\right.$ $\left.3.665 \mathrm{eV}, t_{3}=-0.205 \mathrm{eV}, t_{4}=-0.105 \mathrm{eV}, t_{5}=-0.055 \mathrm{eV}\right)$ and four interlayer hopping terms $\left(t_{p 1}=0.295 \mathrm{eV}, t_{p 2}=\right.$ $\left.0.273 \mathrm{eV}, t_{p 3}=-0.151 \mathrm{eV}, t_{p 4}=-0.091 \mathrm{eV}\right)$. For unbiased bilayer $\mathrm{BP}$, there is an energy splitting of $\Delta \epsilon=1.0 \mathrm{eV}$ between the nonequivalent electrons in the sublayers. This model, which is based on first-principles $G W$ calculations, accurately reproduces the conduction and valence bands for energies $\sim 0.3 \mathrm{eV}$ beyond the gap. For a detailed description of the above TB Hamiltonian we refer the reader to Ref. [10].

We consider two main different sources of disorder in BP: local point defects and long-range disorder potential. Point defects are modeled by elimination of atoms randomly over the whole sample, which can be viewed as vacancies, chemical adsorbates or substitution of other types of atoms which prevent the hopping of electrons to their neighbor 
(a)

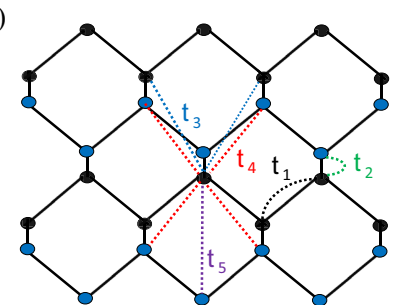

(c)

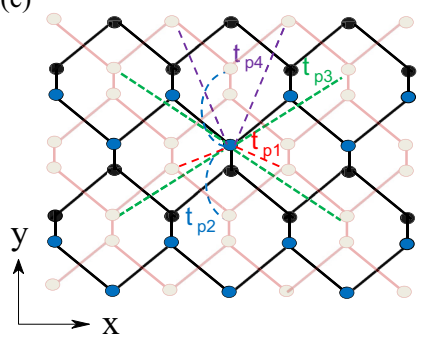

(b)

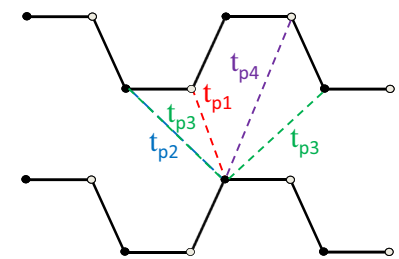

(d)

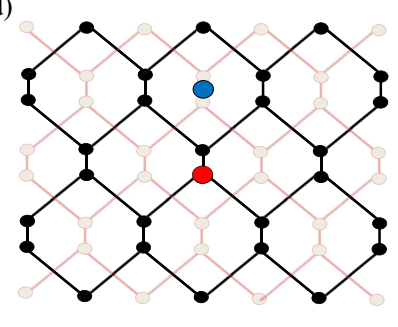

FIG. 1. (Color online) Lattice structure of single-layer and bilayer BP. The relevant hopping terms considered in the Hamiltonian (1) are shown. The red and blue dots in (d) represent a resonant scatterer and a Gaussian center, respectively.

sites [18,28,31-36]. These types of point defects are the so-called resonant scatterers (RS) which provide resonances within the band gap $[18,35,36]$, as confirmed by first-principles calculations which include single vacancies [37,38], adatoms ( $\mathrm{Si}, \mathrm{Ge}, \mathrm{Au}, \mathrm{Ti}, \mathrm{V}$ ) [39], absorption of organic molecules [40], substitutional p-dopants (Te, C) [37] or oxygen bridge-type defects [41].

The long-range disorder potential (LRDP), on the other hand, can account for electron-hole puddles, which are random changes of the local potential due to the inhomogeneous distribution of charge in the sample. Within the TB model, it can be considered as a correlated Gaussian potential [34,42,43], such that the potential at site $i$ follows

$$
v_{i}=\sum_{k} U_{k} \exp \left(-\frac{\left|\mathbf{r}_{i}-\mathbf{r}_{k}\right|^{2}}{2 d^{2}}\right),
$$

where $\mathbf{r}_{k}$ is the position of the $k$ th Gaussian center, which is chosen to be randomly distributed over the centers of the projected lattice on the surface, $U_{k}$ is the amplitude of the potential at the $k$ th Gaussian center, which is uniformly random in the range $[-\Delta, \Delta]$, and $d$ is interpreted as the effective potential radius. Typical values of $\Delta$ and $d$ used in our model are $\Delta=5 \mathrm{eV}$ and $d=5 a$ for LRDP $[18,34,43]$. Here $a \approx 2.216 \AA$ is the atomic distance of two nearest neighbors within the same plane. The origin of LRDP could be screened charged impurities on the substrate [44-47] or surface corrugations such as ripples and wrinkles [48,49]. Although LRDP does not introduce resonances in the spectrum, they lead to a uniform increase of states in the gap [18]. The amount of resonant scatterers and Gaussian centers are quantified by $n_{x}$ and $n_{c}$, respectively, which are the probability for a scatterer or Gaussian center to exist.

The excitation spectrum and screening of single- and bilayer BP are calculated by using the TBPM [28-30]. The dynamic polarization function is obtained from the Kubo

formula [50]:

$$
\Pi(\mathbf{q}, \omega)=\frac{i}{V} \int_{0}^{\infty} d \tau e^{i \omega \tau}\langle[\rho(\mathbf{q}, \tau), \rho(-\mathbf{q}, 0)]\rangle,
$$

where $V$ denotes the volume (or area in two dimensions) of the unit cell, $\rho(\mathbf{q})$ is the density operator given by

$$
\rho(\mathbf{q})=\sum_{l=1}^{N_{\text {layer }}} \sum_{i} c_{l, i}^{\dagger} c_{l, i} \exp \left(i \mathbf{q} \cdot \mathbf{r}_{l, i}\right),
$$

and the average in (3) is taken over the canonical ensemble. For the case of the single-particle Hamiltonian, Eq. (3) can be written as [29]

$$
\begin{aligned}
\Pi(\mathbf{q}, \omega)= & -\frac{2}{V} \int_{0}^{\infty} d \tau e^{i \omega \tau} \operatorname{Im}\langle\varphi| n_{F}(H) e^{i H \tau} \rho(\mathbf{q}) e^{-i H \tau} \\
& \times\left[1-n_{F}(H)\right] \rho(-\mathbf{q})|\varphi\rangle,
\end{aligned}
$$

where $n_{F}(H)=\frac{1}{e^{\beta(H-\mu)}+1}$ is the Fermi-Dirac distribution operator, $\beta=1 / k_{B} T$ where $k_{B}$ is the Boltzmann constant and $T$ is the temperature, and $\mu$ is the chemical potential. We use units such that $\hbar=1$ and the average in Eq. (5) is performed over a random phase superposition of all the basis states in the real space, i.e., [28,51]:

$$
|\varphi\rangle=\sum_{l, i} a_{l, i} c_{l, i}^{\dagger}|0\rangle,
$$

where the coefficients $a_{l, i}$ are random complex numbers normalized as $\sum_{l, i}\left|a_{l, i}\right|^{2}=1$. We next introduce the time evolution of two wave functions:

$$
\begin{gathered}
\left|\varphi_{1}(\mathbf{q}, \tau)\right\rangle=e^{-i H \tau}\left[1-n_{F}(H)\right] \rho(-\mathbf{q})|\varphi\rangle, \\
\left|\varphi_{2}(\tau)\right\rangle=e^{-i H \tau} n_{F}(H)|\varphi\rangle,
\end{gathered}
$$

which allows us to express the real and imaginary part of the dynamic polarization as

$$
\begin{aligned}
& \operatorname{Re} \Pi(\mathbf{q}, \omega)=-\frac{2}{V} \int_{0}^{\infty} d \tau \cos (\omega \tau) \operatorname{Im}\left\langle\varphi_{2}(\tau)|\rho(\mathbf{q})| \varphi_{1}(\tau)\right\rangle, \\
& \operatorname{Im} \Pi(\mathbf{q}, \omega)=-\frac{2}{V} \int_{0}^{\infty} d \tau \sin (\omega \tau) \operatorname{Im}\left\langle\varphi_{2}(\tau)|\rho(\mathbf{q})| \varphi_{1}(\tau)\right\rangle .
\end{aligned}
$$

The time evolution operator $e^{-i H \tau}$ and Fermi-Dirac distribution operator $n_{F}(H)$ can be obtained from the standard Chebyshev polynomial decomposition [28]. In our calculations, the chemical potential is set to be $\sim 0.1 \mathrm{eV}$ above the edge of conduction band, i.e., $\mu=0.4 \mathrm{eV}$ for single-layer $\mathrm{BP}$ and $0.73 \mathrm{eV}$ for bilayer BP. The temperature is fixed at $T=$ $300 \mathrm{~K}$. We use periodic boundary conditions, and the system size is $8192 \times 8192$ for single-layer $\mathrm{BP}$, and $6000 \times 6000$ for bilayer BP. Since our calculations are performed on samples with more than millions of atomic sites, the numerical results are not sensitive to a particular choice of the disorder distribution [28,29].

Electron-electron interactions are considered within the RPA. The two-dimensional dielectric function for single-layer phosphorene is calculated as

$$
\varepsilon_{1 L}(\mathbf{q}, \omega)=\mathbf{1}-V(q) \Pi(\mathbf{q}, \omega),
$$


where

$$
V(q)=\frac{2 \pi e^{2}}{\kappa q}
$$

is the Fourier component of the Coulomb interaction in two dimensions, in terms of the background dielectric constant $\kappa$. For bilayer BP, the Coulomb interaction has a matrix form,

$$
\tilde{\mathbf{V}}(q)=\left(\begin{array}{ll}
V_{\text {intra }}(q) & V_{\text {inter }}(q) \\
V_{\text {inter }}(q) & V_{\text {intra }}(q)
\end{array}\right)
$$

where $V_{\text {intra }}(q)=V(q)$ as given by Eq. (11), and the interlayer interaction between electrons in different BP layers is $V_{\text {inter }}=$ $F(q) V(q)$ where $F(q)=e^{-q d}$, and $d=0.5455 \mathrm{~nm}$ is the interlayer distance. From this we can express the dielectric tensor for bilayer BP, including the intralayer and interlayer contributions, as

$$
\varepsilon_{2 L}(\mathbf{q}, \omega)=\left(\begin{array}{cc}
\varepsilon_{1 L}(\mathbf{q}, \omega) & -V(q) F(q) \Pi(\mathbf{q}, \omega) \\
-V(q) F(q) \Pi(\mathbf{q}, \omega) & \varepsilon_{1 L}(\mathbf{q}, \omega)
\end{array}\right) .
$$
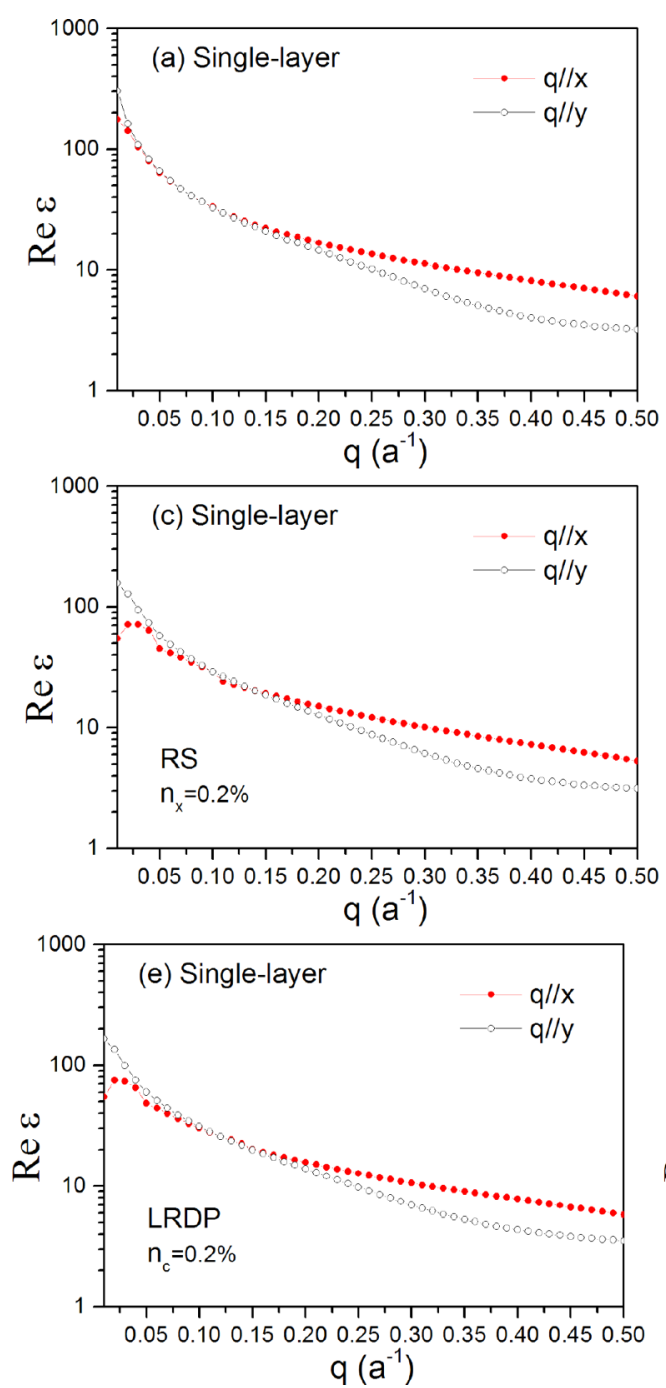

In the next sections we use the polarization and dielectric functions defined here to study the effect of disorder on the screening properties and the collective plasmon modes of BP. To simplify the notation, we use $\varepsilon(\mathbf{q}, \omega)$ to represent $\varepsilon_{1 L}(\mathbf{q}, \omega)$ for single-layer BP and the determinant of the dielectric tensor $\left|\varepsilon_{2 L}(\mathbf{q}, \omega)\right|$ for bilayer BP.

\section{DIELECTRIC SCREENING}

We start by studying the effect of disorder on the screening properties. In Fig. 2, we plot the static dielectric function $\varepsilon(\mathbf{q}, 0)$ of single- and bilayer BP. There is a clear dependence of the static dielectric function with the direction of momentum, showing a different behavior for $\mathbf{q}$ along the zigzag $(X)$ or armchair $(Y)$ directions. For the pristine case, our numerical results for the momentum dependence of the static dielectric function of single-layer phosphorene, Fig. 2(a), agree with the analytic low energy model presented in Ref. [22]. The present calculation within the TBPM method allows us to extend the
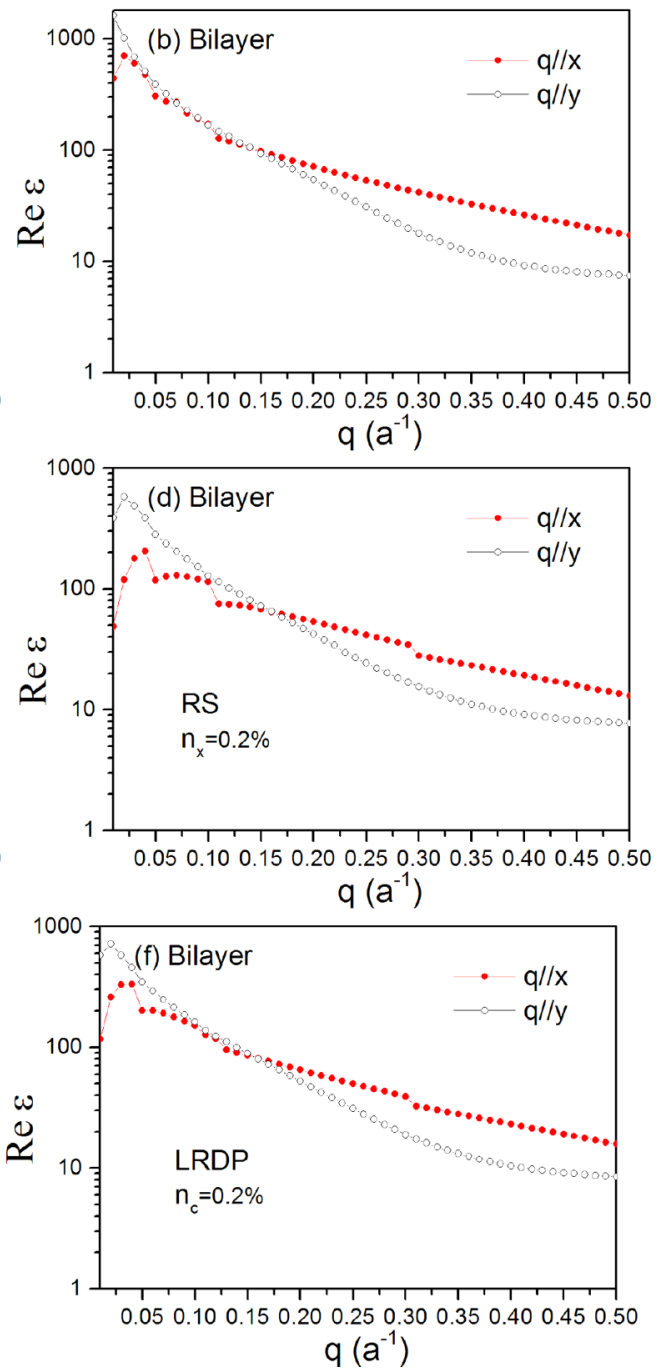

FIG. 2. (Color online) Static dielectric function of single- (left) and bilayer (right) BP. The chemical potential is set to be $\mu=0.4 \mathrm{eV}$ for single layer and $0.73 \mathrm{eV}$ for bilayer. The temperature is fixed as the room temperature $T=300 \mathrm{~K}$. Plots (a) and (b) correspond to pristine $\mathrm{BP}$, (c) and (d) correspond to samples with resonant scatterers originated from a concentration of $n_{x}=0.2 \%$ vacant atoms, and (e) and (f) correspond to samples with long-range disorder potential with a concentration of $n_{c}=0.2 \%$ Gaussian centers. 

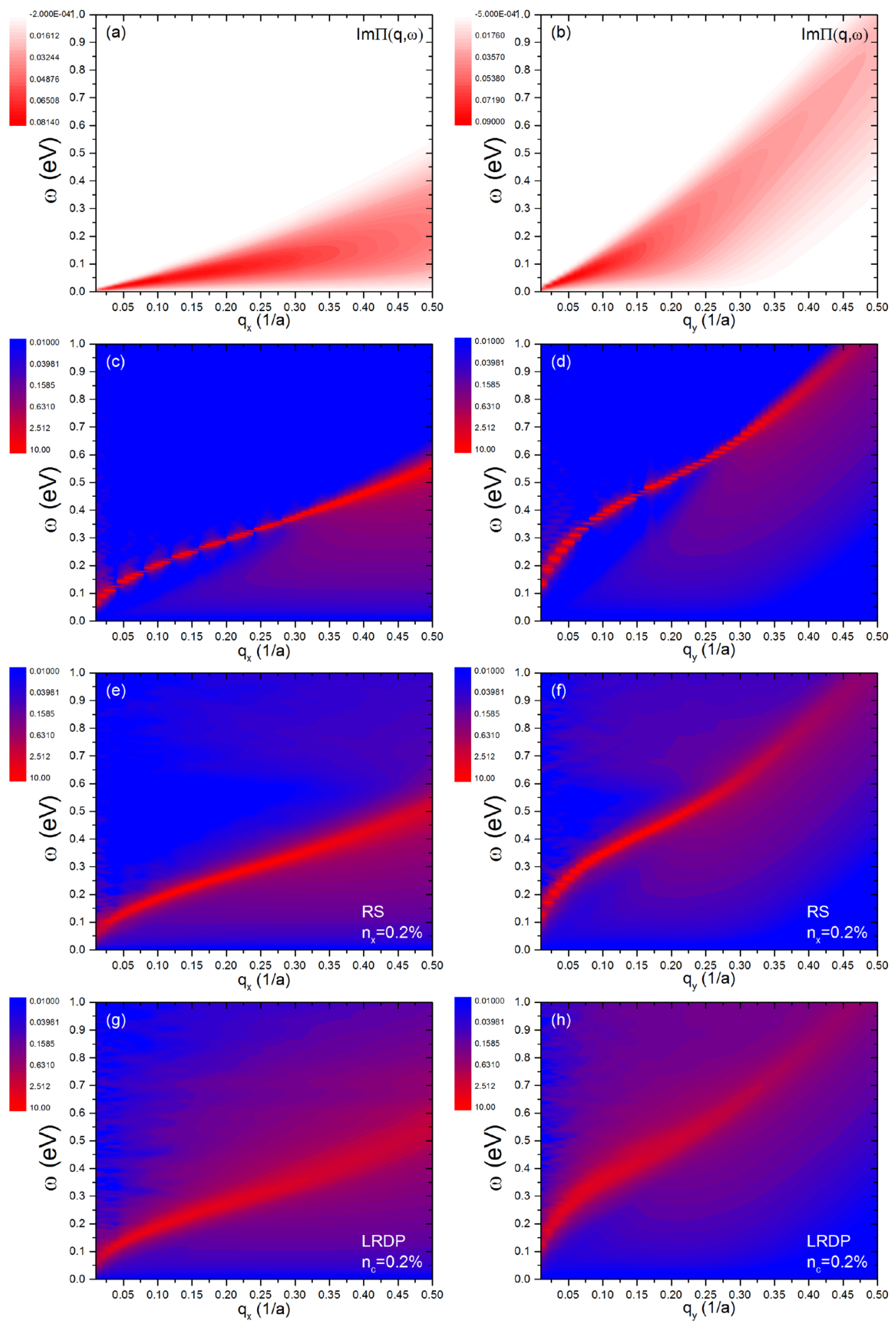

FIG. 3. (Color online) Electron-hole continuum as defined from the noninteracting polarization function (a) and (b), and energy loss functions (c)-(h) of single-layer BP along zigzag (left) and armchair (right) directions. The simulations are done for (a)-(d) pristine BP, (e) and (f) samples with resonant scatterers, and (g) and (h) samples with long-range disorder potentials. All the results are calculated at $T=300 \mathrm{~K}$. We notice that the apparent discretization of the spectrum in some of the plots is an artifact due to finite size limitations in our calculations.

calculation of Ref. [22] to larger wave vectors and to consider realistic impurities in the sample. We notice that the size of the lattices used in our simulations determines the range of wave vectors for our numerical results, which is $q \geqslant 0.01 a^{-1}$. We do not perform calculations with momentum transfer smaller than $q=0.01 a^{-1}$ since, in order to preserve the numerical accuracy for $q \rightarrow 0$, an extremely large sample size $N \rightarrow \infty$ is required. 
In both single- and bilayer BP, pointlike resonant scatterers lead to the creation of midgap states inside the gap [18]. These states are quasilocalized around the center of the impurity. Therefore, the typical divergence of the dielectric function at $q \rightarrow 0$, characteristic of metallic behavior, is reduced for BP crystals with this kind of disorder, as it can be seen in Figs. 2(c) and 2(d). A similar result for the dielectric function is obtained for LRDP which can be associated with the existence of electron-hole puddles in the sample. A drop of the dielectric function at small $q$ appears also in disordered graphene with chemical absorbers [30]. The effect of disorder on the static dielectric function is negligible at short wavelengths (large $q$ vectors).

\section{ENERGY LOSS FUNCTION AND PLASMONS}

In this section we study the collective modes of the system, which are defined by the zeros of the dielectric function $\varepsilon(\mathbf{q}, \omega)$ for single-layer BP (or zeros of the dielectric tensor determinant for bilayer BP). The dispersion relation of the plasmon modes is defined from $\operatorname{Re} \varepsilon\left(\mathbf{q}, \omega_{p l}\right)=0$, which leads to poles in the energy loss function $-\operatorname{Im} 1 / \varepsilon(q, \omega)$, that can be measured by means of electron energy loss spectroscopy (EELS) [52]. In the clean and zero temperature limit, plasmon modes are fully coherent unless their dispersion enters in the electron-hole continuum, which is the region of the energy-momentum space where electron-hole pairs can exist. The electron-hole continuum is defined as the region of the spectrum where $\operatorname{Im} \Pi(\mathbf{q}, \omega) \neq 0$, and we show it for singlelayer and bilayer phosphorene in the top panels of Figs. 3 and 6 , respectively.
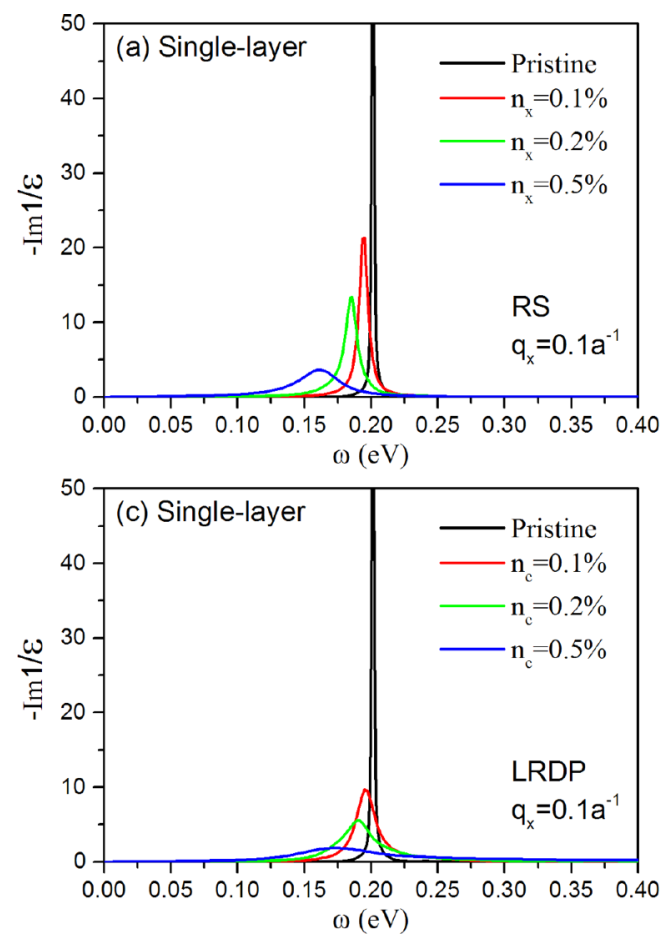

\section{A. Single-layer BP}

For single-layer BP, our numerical results for the loss function are plotted in Fig. 3 and show anisotropic plasmon modes along the zigzag and armchair directions, both following a low energy $\sqrt{q}$ dependence at small momentum transfer. This is due to the paraboloidal band dispersion of BP at low energies, and it is consistent with the results for the excitation spectrum of BP within the $\mathbf{k} \cdot \mathbf{p}$ approximation [22]. The present method allows us to study the spectrum at higher energies and wave vectors, including also the effect of disorder in the sample. The sharpness of the plasmon dispersion is mainly affected by two factors from a numerical point of view: the size of the sample which provides a finite number of energy eigenstates, and the energy resolution which is inversely proportional to the total number of time steps counted during the wave propagation.

In the presence of disorder, there is a broadening and redshift of the plasmon modes, as can be seen in Figs. 3(e) and 3(f) for single-layer samples with resonant scatterers, and in Figs. 3(g) and 3(h) for long-range disorder potential. For a fixed wave vector, the effect of disorder is better seen by the loss function shown in Fig. 4 for $q=0.1 a^{-1}$ and different concentrations of disorder. Notice that the presence of LRDP induces a stronger damping of the plasmon, as compared to point defects. This is seen by comparing the broadening of the peaks of the top and bottom panels of Fig. 4. Since plasmons are collective electronic oscillations induced by long-range Coulomb interaction, it is expected that long-range disorder affects more the coherence of these modes than local scatterers as vacancies, which are relevant at short length scales.

Figure 4 also shows that, for a given wavelength, the two kinds of disorder considered in our simulations lead to a shift of
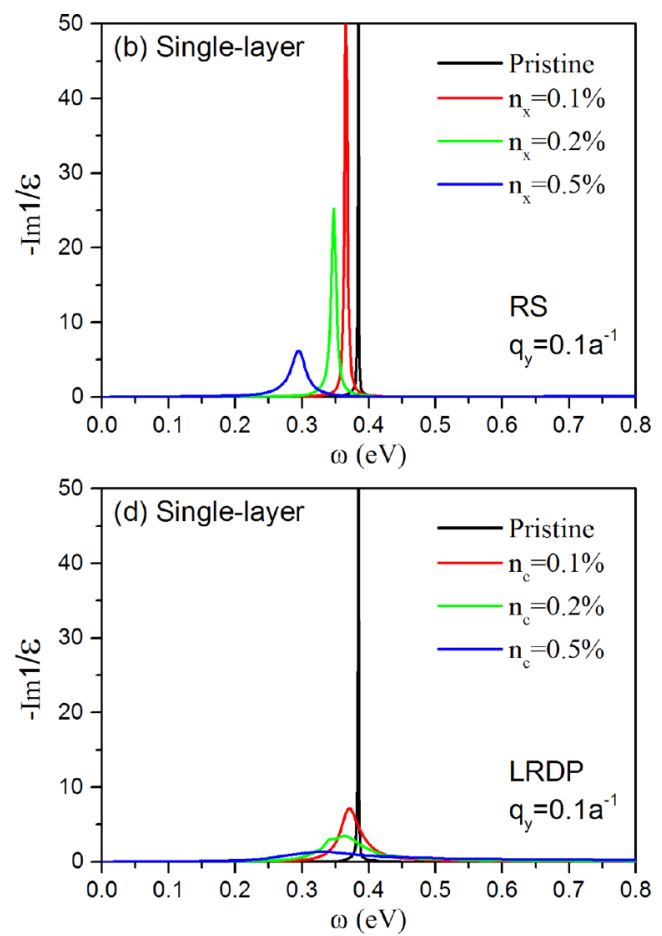

FIG. 4. (Color online) Energy loss functions of single-layer BP along zigzag (left) and armchair (right) directions. Panels (a) and (b) compare the loss function of pristine single-layer BP with that of samples with different concentrations of point defects. Panels (c) and (d) correspond to LRDP. All the results are calculated at $T=300 \mathrm{~K}$. 
the plasmon resonance towards lower energies. This effect can be understood from a perturbative point of view, by using the disordered averaged response function introduced by Mermin in the context of a 2DEG with a parabolic band dispersion [53], and which has been recently used to study the effect of weak disorder on the plasmon dispersion of spin-polarized graphene [54]. In the weak disorder limit, and assuming for simplicity an isotropic parabolic band dispersion, the disordered averaged response function has the form $[53,55]$,

$\Pi_{\mathrm{imp}}(\mathbf{q}, \omega) \approx-N(0)\left(1-\frac{\omega}{\sqrt{\left(\omega+i / \tau_{\mathrm{el}}\right)^{2}-v_{F}^{2} q^{2}}-i / \tau_{\mathrm{el}}}\right)$,

where $\tau_{\mathrm{el}}$ is the elastic lifetime of the momentum eigenstates of the disordered system, and $N(0)=g m_{b} / 2 \pi$ is the density of states (per unit area) at the Fermi energy, where $g=2$ is the spin degeneracy, $m_{b}$ is the effective mass, and $v_{F}$ is the Fermi velocity. In the presence of disorder the dispersion relation of the plasmon will have, even in the low energy and long wavelength limit, an imaginary part, due to finite damping of the plasmon. Within this approximation, it is possible to obtain an analytical expression for the plasmon dispersion by substituting (13) into (10) and then finding the zeros of the dielectric function, with the approximate solution,

$$
\omega_{p l}(q) \approx \frac{q+4 r_{s} k_{F}}{q+8 r_{s} k_{F}}\left(\sqrt{v_{F}^{2} q\left(q+8 r_{s} k_{F}\right)-1 / \tau_{\mathrm{el}}^{2}}-i / \tau_{\mathrm{el}}\right),
$$
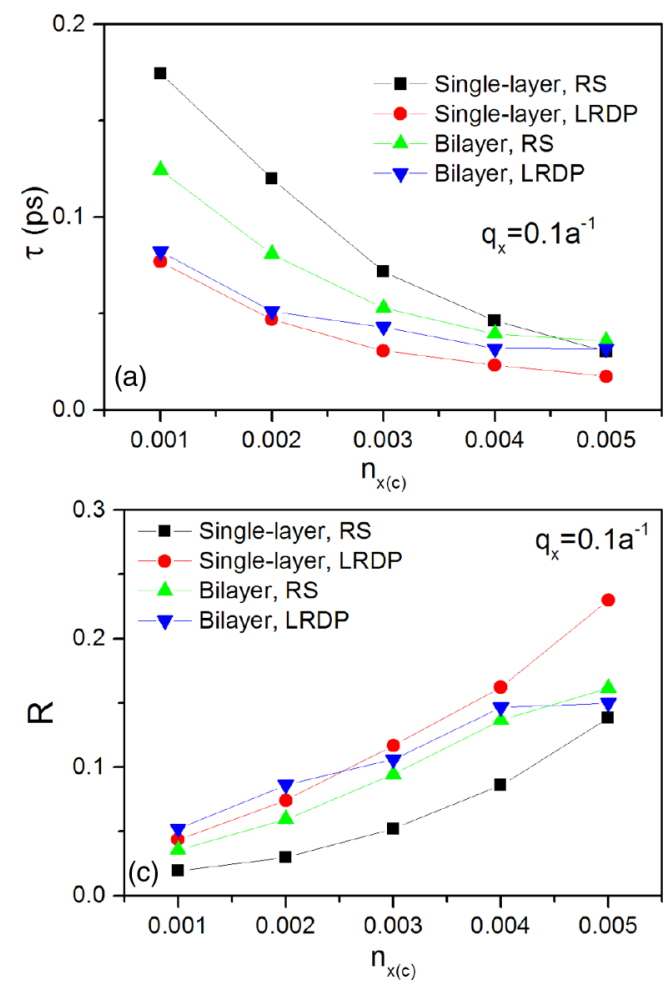

where $r_{s}=2 m_{b} e^{2} / \kappa k_{F}$ is the dimensionless interaction parameter of a 2DEG and $k_{F}$ is the Fermi wave vector. Equation (14) reduces to the standard plasmon dispersion in the clean $\left(1 / \tau_{\mathrm{el}} \rightarrow 0\right)$ limit. We notice here that the presence of impurities limits the dispersion of collective plasmon modes for length scales longer than the mean-free path, defining a minimal wave vector below which the plasmon mode is not well defined. The existence of such a length scale has been discussed, in the context of graphene, in Ref. [54]. For the case of interest here such infrared cutoff takes the form $q_{\min }=-4 r_{s} k_{F}+\sqrt{16 r_{s}^{2} k_{F}^{2}+1 / \tau_{\mathrm{el}}^{2} v_{F}^{2}}$, below which plasmon modes do not exist. In our calculations, since we consider a finite sample in real space, the wave vectors in momentum space are much larger than the cutoff values of $q_{\mathrm{min}}$. For example, for single-layer BP with $\mu=0.4 \mathrm{eV}$, the numerical results of the Fermi wave vector, effective masses, and Fermi velocity along the zigzag direction are $k_{x}=0.256 a^{-1}, m_{x}=$ $0.86482 m_{e}$, and $v_{F, x}=1.1636 \times 10^{5} \mathrm{~m} / \mathrm{s}$ and along the armchair direction are $k_{y}=0.116 a^{-1}, m_{y}=0.20536 m_{e}$, and $v_{F, y}=3.0621 \times 10^{5} \mathrm{~m} / \mathrm{s}$, respectively. The free lifetime of the electron can be extracted from the time evolution of the diffusion coefficient within the TBPM (for details about the numerical method we refer to Ref. [30]). Using random resonant scatters with impurity concentration $n_{x}=0.2 \%$ as an example, the anisotropic free lifetime is $\tau_{x} \approx 740 \mathrm{fs}$ and $\tau_{y} \approx 1000 \mathrm{fs}$, extracted from the transport calculations in Ref. [30]. Therefore the infrared cutoff for the plasmon mode associated with the finite mean-free path of the disordered sample is $q_{\min , x} \approx 9 \times 10^{-9} a^{-1}$ and $q_{\min , y} \approx 3 \times 10^{-9} a^{-1}$, both of them much smaller than the minimal wave vector
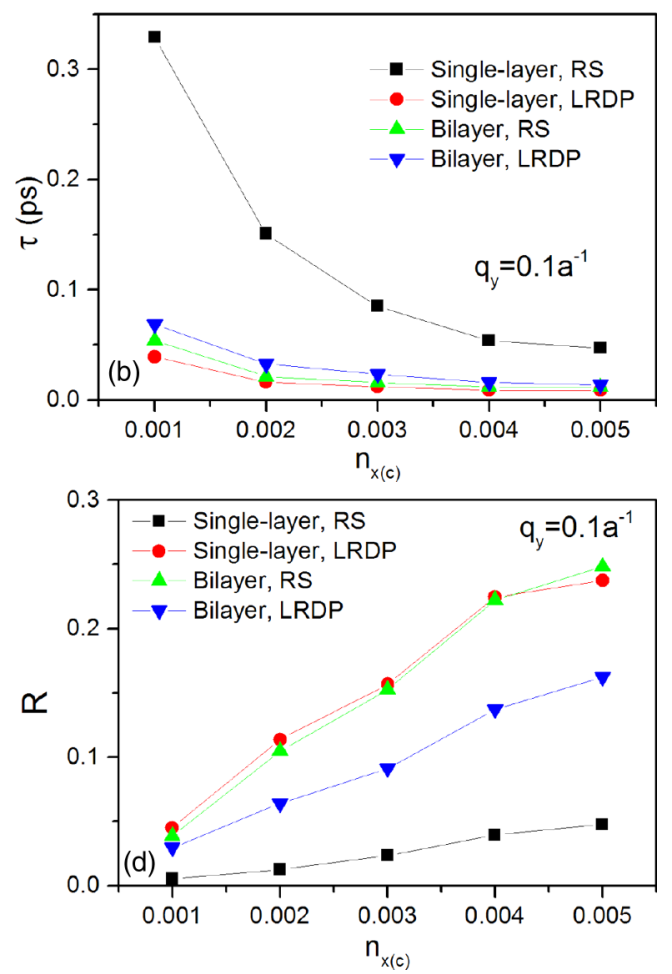

FIG. 5. (Color online) Effect of disorder on plasmon losses. Panels (a) and (b) show the plasmon lifetime, and panels (c) and (d) show the corresponding damping rates (inverse quality factor). The results for bilayer BP samples correspond to the in-phase $\sim \sqrt{q}$ plasmon mode (see text). 

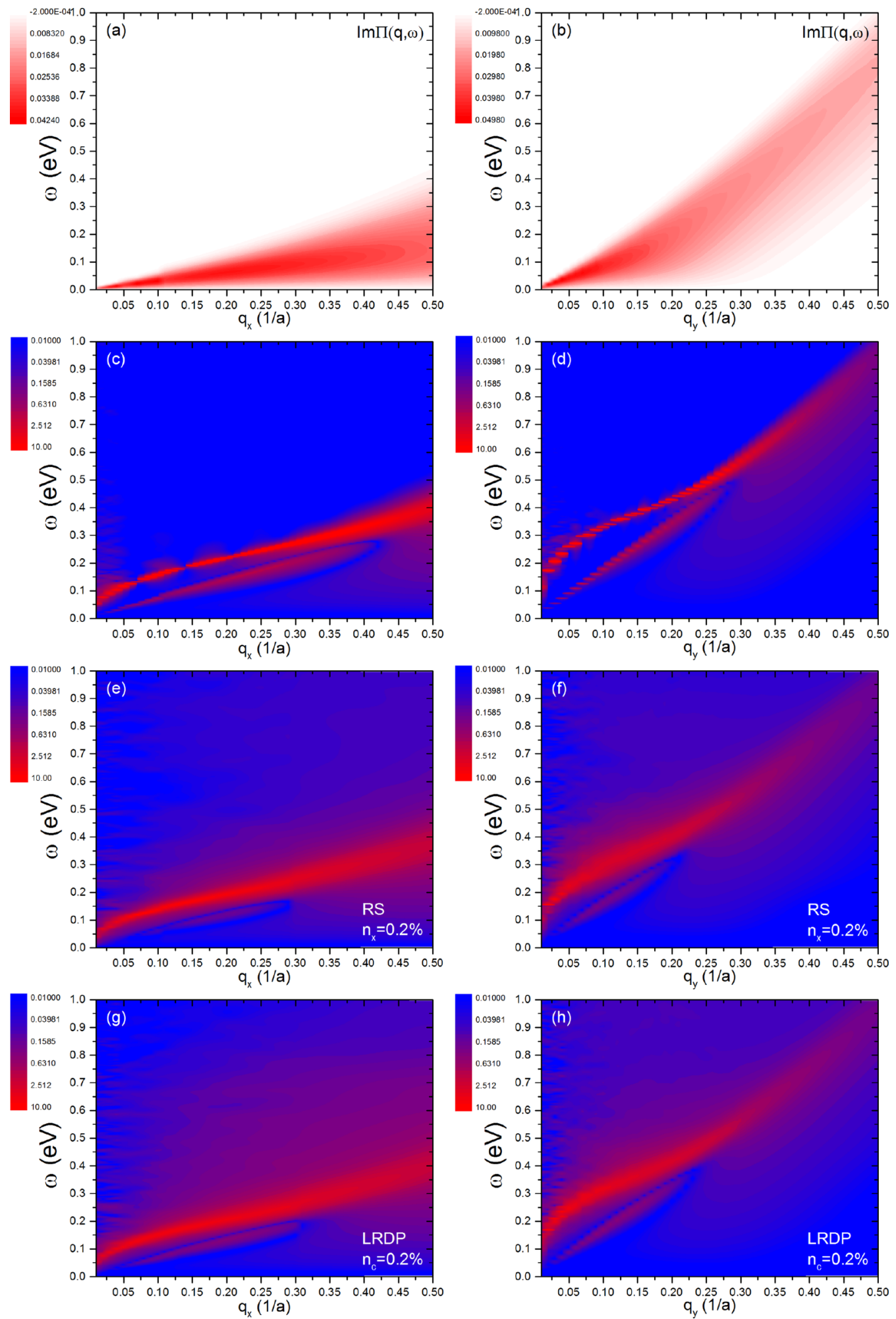

FIG. 6. (Color online) Electron-hole continuum as defined from the noninteracting polarization function (a) and (b), and energy loss functions (c)-(h) of bilayer BP along zigzag (left) and armchair (right) directions. The simulations are done for (a)-(d) pristine bilayer BP, (e) and (f) samples with resonant scatterers, and (g) and (h) samples with long-range disorder potentials. All the results are calculated at $T=300 \mathrm{~K}$.

$|q|=0.1 a^{-1}$ that can be considered here, due to finite size of the simulated samples.

The next step in our analysis is to quantify the effect of disorder on plasmon losses. The physical observability of a damped collective mode depends on the sharpness of the resonance peak, and it is defined as a dimensionless inverse quality factor $\mathcal{R}=\gamma / \omega_{p l}$, where the damping rate $\gamma$ of the mode, which is proportional to $\operatorname{Im} \Pi\left(\mathbf{q}, \omega_{p l}\right)$, is given 

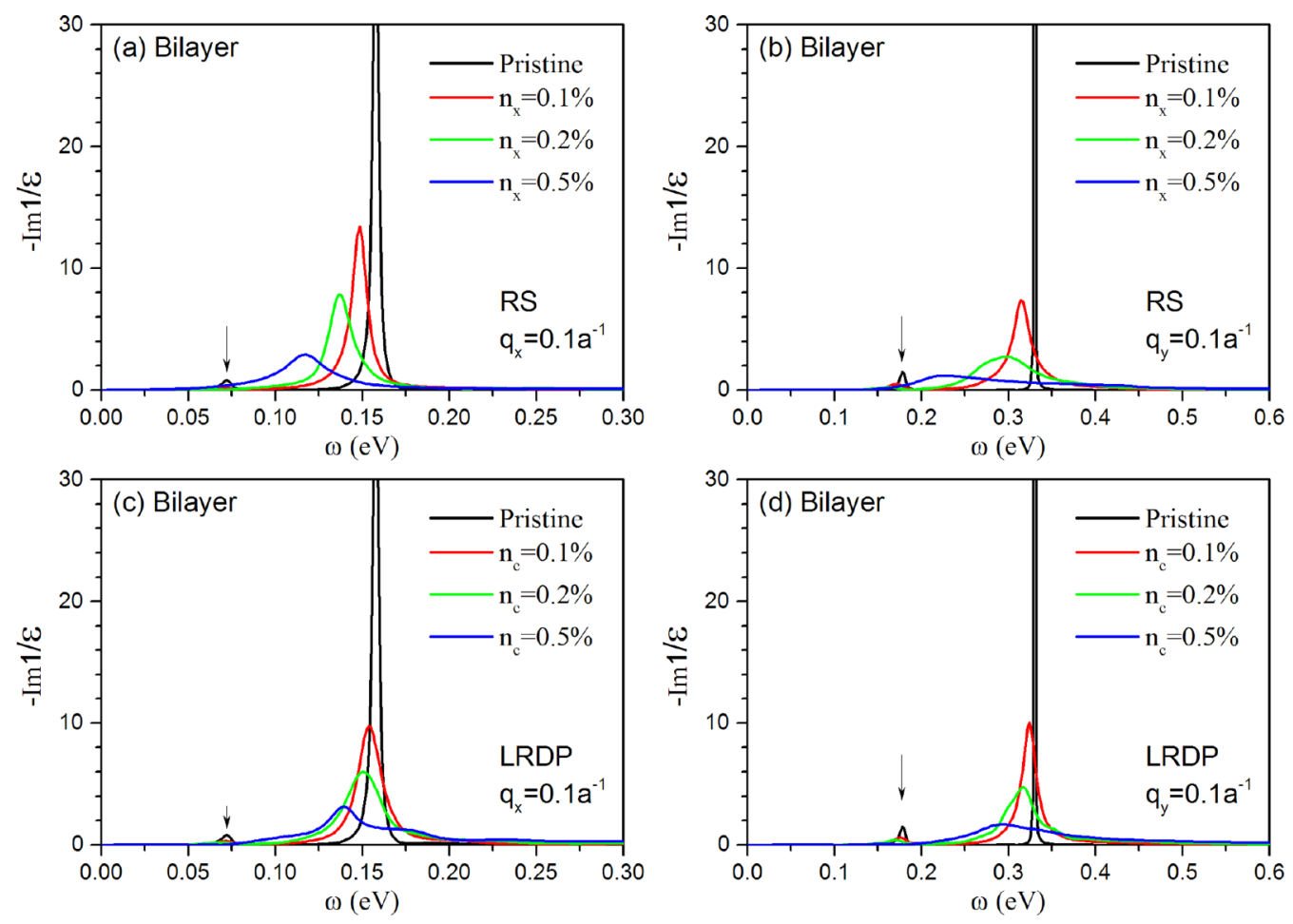

FIG. 7. (Color online) Energy loss functions of bilayer BP along zigzag (left) and armchair (right) directions. Panels (a) and (b) compare the loss function of pristine bilayer BP with that of samples with different concentrations of point defects. Panels (c) and (d) correspond to LRDP. All the results are calculated at $T=300 \mathrm{~K}$. The peak marked by arrows in each panel correspond to the out-of-phase $\omega_{-}(q) \sim q$ plasmon (see text).

by

$$
\gamma=\frac{\operatorname{Im} \Pi\left(\mathbf{q}, \omega_{p l}\right)}{\left.\frac{\partial}{\partial \omega} \operatorname{Re} \Pi(\mathbf{q}, \omega)\right|_{\omega=\omega_{p l}}} .
$$

The plasmon lifetime $\tau$ is calculated via the inverse quality factor as $\tau=1 / \mathcal{R} \omega_{p l}$. We have calculated numerically $\mathcal{R}(\gamma)$ and $\tau$, and the results are shown in Fig. 5. We first notice that, for a given concentration of impurities and for a given wavelength, the plasmon rate is anisotropic. In fact, our results show that plasmons dispersing in the $y$ direction (armchair) are more efficiently damped than plasmons dispersing in the $x$ (zigzag) direction, which have a larger lifetime. Second, as discussed above, we find that short-range resonant scatterers like point defects cause fewer losses in the plasmon coherence than LRDP.

\section{B. Bilayer BP}

In the following we present results for bilayer BP, considered as two phosphorene layers coupled by interlayer hopping (see Fig. 1), and interacting via long-range Coulomb potential, as explained in Sec. II. Our results for the loss function are shown in Fig. 6. The first thing to notice is the existence of two collective modes in the spectrum, as it is usual in bilayer systems [23,56-58]. One is the classical $\omega_{+}(q \rightarrow 0) \sim \sqrt{q}$ plasmon mode, which has its counterpart in single-layer samples (see Fig. 3). This mode corresponds to a collective excitation of the electron liquid in which the carriers of both layers oscillate in-phase. In addition to this plasmon, in Fig. 6 we observe the existence of an additional mode in the excitation spectrum dispersing below the $\omega_{+}$ plasmon. This mode, with a low energy linear dispersion relation, $\omega_{-}(q \rightarrow 0) \sim q$, is characteristic of bilayer $2 \mathrm{DEG}$ systems and corresponds to a collective oscillation in which the carrier density in the two layers oscillates out-of-phase. The existence of this mode is also clear in Fig. 7, where it can be seen that, besides the peak in the loss function corresponding to the in-phase $\omega_{+}$plasmon, there is a second resonance at lower energies (with less spectral weight) which corresponds to the $\omega_{-} \sim q$ plasmon (indicated by black arrows). The $\omega_{-}$ mode is much weaker as compared to the $\omega_{+}$mode because of the short distance between the two layers, which complicates the out-of-phase density oscillation. In Ref. [23], the two modes discussed above have been also obtained by using a general low energy model of anisotropic double-layer systems. We notice here that, since they study bilayer systems in which the two layers are separated by more than $5 \mathrm{~nm}$ [23], their results show a $\omega_{-}$mode that is more coherent and dispersing than the one obtained in our calculations. As a matter of fact, the dispersion relation of the out-of-phase mode is proportional to the layer separation, $\omega_{-}(q) \sim d^{1 / 2} q$, where $d$ is the interlayer distance. Here we restrict our study to real bilayer phosphorene, in which the two layers are separated by $d=0.524 \mathrm{~nm}$, and interlayer hopping of electrons is allowed. As a consequence, the $\omega_{-}$mode obtained in our simulations for bilayer BP disperses with a smaller velocity than the mode of Ref. [23].

We also observe that, as in the single-layer case, both modes are damped due to disorder. The characteristics of the damping of the in-phase $\omega_{+} \sim \sqrt{q}$ mode can be understood from the previous analysis for single-layer BP. Losses are 

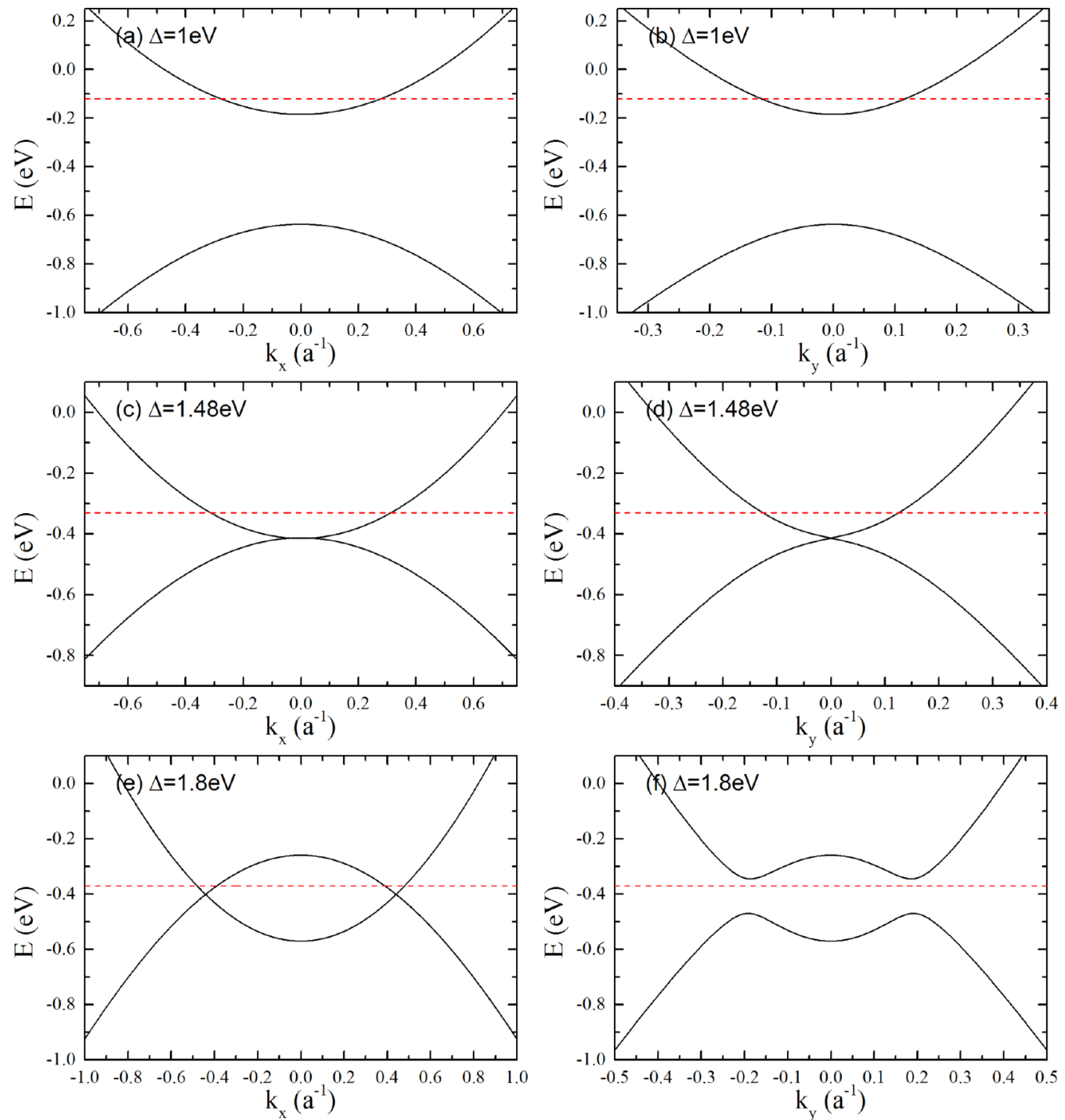

FIG. 8. (Color online) Low energy band structure of bilayer BP in the presence of a perpendicular electric field, for different biased potential energy between top and bottom layers. The red dashed line in each panel indicates the position of the chemical potential in the calculation of the plasmon modes.

stronger in the out-of-phase $\omega_{-}$plasmon, whose coherence basically vanishes due to disorder. The reasons for this are twofold. On the one hand, the spectral weight of the $\omega_{-}$mode is considerably weaker than the $\omega_{+}$plasmon. On the other hand, the $\omega_{-}$mode disperses in the $\omega-q$ spectrum very close to the electron-hole continuum, whose threshold can be reached due to disorder (and thermal) broadening of the plasmon peak, with the consequent Landau damping of the mode.

\section{Biased bilayer BP}

In this section we include in our calculations the presence of a perpendicular electric field applied to the bilayer BP. This technique can be used to manipulate the electronic band structure of the system, and it has been proposed recently as an appropriate way to drive a normal to topological phase in this material $[59,60]$. Here we are interested in studying the effect of an electric field on the dispersion relation of plasmons. For this aim, we introduce a biased on-site potential difference $\Delta$ between the two layers as described in Ref. [61], and consider three representative cases, as shown in Fig. 8: $\Delta=1 \mathrm{eV}$, for which the band gap is reduced, $\Delta=1.4 \mathrm{eV}$, for which the gap completely closes, and $\Delta=1.8 \mathrm{eV}$, for which the conduction and valence bands are overlapped, corresponding to the topological phase discussed in Refs. [59,60]. It is interesting to notice that, for this last case, the band structure of bilayer BP presents Dirac-like cones for the dispersion in the $k_{x}$ direction [Fig. 8(e)], and the spectrum is gapped in the $k_{y}$ direction [Fig. 8(f)]. For $\Delta=1 \mathrm{eV}$ and $1.4 \mathrm{eV}$, the chemical potential is chosen to be about $0.1 \mathrm{eV}$ above the edge of the conduction band, to better compare with the results of unbiased bilayer BP of Fig. 6.

Our results show that the velocity of the plasmon mode increases with the biased voltage, as it can be seen from the evolution of the excitation spectrum with the applied $\Delta$ in Fig. 9. This suggests the possibility of tune the plasmonic properties of this material with a perpendicular electric field. Interestingly, when the applied electric field exceeds the 

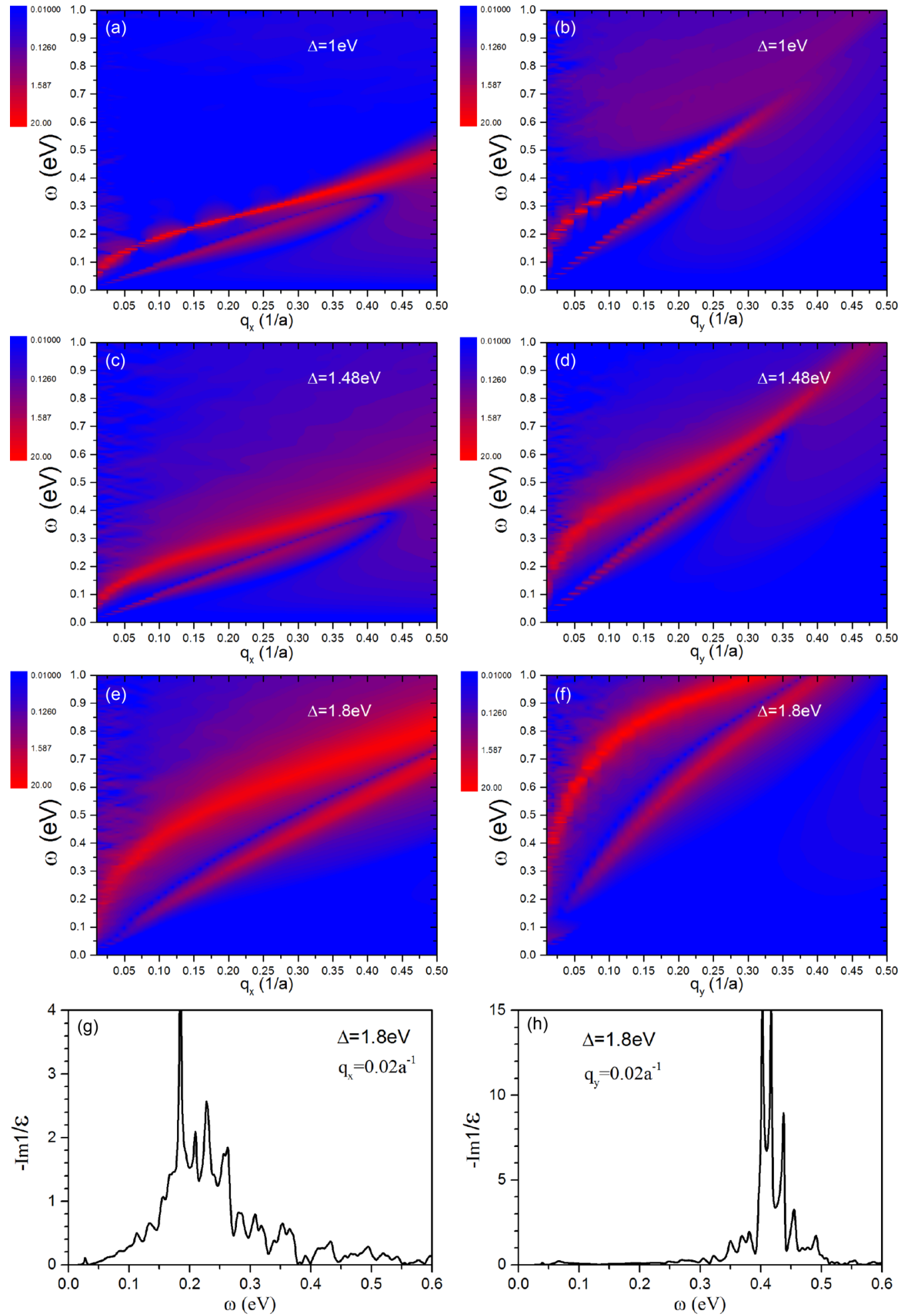

FIG. 9. (Color online) Energy loss functions of biased bilayer BP along zigzag (left) and armchair (right) directions. The strength of the biased potential is given in each panel.

critical field and enters in the topological phase, the plasmons are more coherent, as it can be inferred by looking at the strength of the modes in the density plots of Fig. 9. Furthermore, the plasmon dispersion is ungapped along the $q_{x}$ direction, whereas the presence of a gap in the electronic band structure in the $\Gamma-Y$ direction leads to a gapped collective mode along $q_{y}$, as it can be seen by looking at Fig. 9(f) in the low energy and small wave-vector region. We notice again that, due to the finite size nature of our simulations, our results cannot reach the $q \rightarrow 0$ limit, for which we would need to consider an infinite sample. However, the existence of a gap in the plasmon spectrum $q_{y}$ direction is already 
clear in the present calculation for $\Delta=1.8 \mathrm{eV}$. Notice that the peculiar band structure in the topological phase, with Dirac-like band crossings in one direction and gap in the other direction, leads to a rich excitation spectrum with numerous peaks corresponding to the plasmon collective excitations and to the enhanced optical transitions due to the appearance of Van Hove singularities in the spectrum. It is important to notice that a possible route to achieve the exotic physical properties of multilayer BP in this topological regime is by in situ deposition of potassium atoms, that induces doping and a vertical electric field across the multilayer sample, as it has been reported experimentally [62].

\section{CONCLUSIONS}

In conclusion, we have studied the effect of disorder in the excitation spectrum of single-layer and bilayer BP. The band structure has been calculated with an accurate tight-binding model which is valid within $\sim 0.3 \mathrm{eV}$ beyond the gap. The dynamical polarization function has been calculated with the Kubo formula, and from this, the energy loss function has been obtained within the RPA. We have found that disorder leads to a redshift of the plasmon resonance. This effect has been discussed from a perturbative point of view, within the framework of the disordered averaged response function [53].

The different kinds of disorder that have been analyzed show that, for the same concentration of impurities, LRDP leads to stronger damping rates as compared to local point defects. Such an effect is understood from the intrinsic long wavelength nature of the plasmon oscillations, induced by long-range Coulomb interaction. Therefore resonant scatterers as point vacancies, which modify the electronic properties at very short length scales, are less effective inducing losses of the plasmon modes than LRDP.

The spectrum of bilayer BP presents two plasmon modes. One mode in which the carrier density in the two planes oscillates in-phase, with a dispersion $\omega_{+}(q) \sim \sqrt{q}$, which is the counterpart of the standard plasmon in single-layer $\mathrm{BP}$, and one mode in which the carriers in the two layers oscillate out-of-phase, with a liner dispersion relation at low energies, $\omega_{-}(q) \sim q$. The coherence of the $\omega_{-}$mode is more dramatically affected by disorder.

Finally, we have studied the effect of a perpendicular electric field in the excitation spectrum of bilayer BP. We have shown that the dispersion of the collective modes can be tuned by the application of such biased field. Furthermore, we have shown that, beyond some critical field, the bilayer BP enters in a topological phase with Dirac-like crossing of bands in one direction and gapped in the other direction. As a consequence, the excitation spectrum is highly rich in this limit, with highly coherent plasmon modes which are gapped in the $q_{y}$ direction of the spectrum. In summary, our results show a highly anisotropic excitation spectrum of single-layer and bilayer BP. The use in black phosphorus of recently developed techniques to excite plasmons in graphene [63-66], such as gated microribbon arrays, nanodots and antidots arrays, periodic deformation by means of surface acoustic waves or by elastomeric substrate, etc., could exploit the plasmonic properties of black phosphorus for future optoelectronic applications.

\section{ACKNOWLEDGMENTS}

Support from the Stichting Fundamenteel Onderzoek der Materie (FOM) and the Netherlands National Computing Facilities foundation (NCF) is acknowledged. S.Y. and M.I.K. thank the European Research Council Advanced Grant program (Contract No. 338957) for financial support. The research has also received funding from the European Union Seventh Framework Programme under Grant Agreement No. 604391 Graphene Flagship. R.R. acknowledges support from the European Research Council Advanced Grant (Contract No. 290846).
[1] L. Li, Y. Yu, G. J. Ye, Q. Ge, X. Ou, H. Wu, D. Feng, X. H. Chen, and Y. Zhang, Nat. Nanotech. 9, 372 (2014).

[2] H. Liu, A. T. Neal, Z. Zhu, Z. Luo, X. Xu, D. Tománek, and P. D. Ye, ACS Nano 8, 4033 (2014).

[3] F. Xia, H. Wang, and Y. Jia, Nat. Commun. 5, 4458 (2014).

[4] S. P. Koenig, R. A. Doganov, H. Schmidt, A. H. Castro Neto, and B. Özyilmaz, Appl. Phys. Lett. 104, 103106 (2014).

[5] A. Castellanos-Gomez, L. Vicarelli, E. Prada, J. O. Island, K. L. Narasimha-Acharya, S. I. Blanter, D. J. Groenendijk, M. Buscema, G. A. Steele, J. V. Alvarez et al., 2D Materials 1, 025001 (2014).

[6] L. Li, G. J. Ye, V. Tran, R. Fei, G. Chen, H. Wang, J. Wang, K. Watanabe, T. Taniguchi, L. Yang et al., Nature Nanotechnology 10, 608 (2015).

[7] A. Morita, Appl. Phys. A 39, 227 (1986).

[8] X. Ling, H. Wang, S. Huang, F. Xia, and M. S. Dresselhaus, Proc. Natl. Acad. Sci. USA 112, 4523 (2015).
[9] J. Qiao, X. Kong, Z.-X. Hu, F. Yang, and W. Ji, Nat. Commun. 5, 4475 (2014).

[10] A. N. Rudenko and M. I. Katsnelson, Phys. Rev. B 89, 201408 (2014).

[11] J. Guan, Z. Zhu, and D. Tománek, Phys. Rev. Lett. 113, 046804 (2014).

[12] X. Peng, Q. Wei, and A. Copple, Phys. Rev. B 90, 085402 (2014).

[13] V. Tran, R. Soklaski, Y. Liang, and L. Yang, Phys. Rev. B 89, 235319 (2014).

[14] D. Çakır, H. Sahin, and F. M. Peeters, Phys. Rev. B 90, 205421 (2014).

[15] T. Low, A. S. Rodin, A. Carvalho, Y. Jiang, H. Wang, F. Xia, and A. H. Castro Neto, Phys. Rev. B 90, 075434 (2014).

[16] T. Low, M. Engel, M. Steiner, and P. Avouris, Phys. Rev. B 90, 081408 (2014).

[17] P. Li and I. Appelbaum, Phys. Rev. B 90, 115439 (2014).

[18] S. Yuan, A. N. Rudenko, and M. I. Katsnelson, Phys. Rev. B 91, 115436 (2015). 
[19] A. S. Rodin, A. Carvalho, and A. H. Castro Neto, Phys. Rev. Lett. 112, 176801 (2014).

[20] M. Elahi, K. Khaliji, S. M. Tabatabaei, M. Pourfath, and R. Asgari, Phys. Rev. B 91, 115412 (2015).

[21] R. Roldán, A. Castellanos-Gomez, E. Cappelluti, and F. Guinea, J. Phys. Condens. Matter 27, 313201 (2015).

[22] T. Low, R. Roldán, H. Wang, F. Xia, P. Avouris, L. M. Moreno, and F. Guinea, Phys. Rev. Lett. 113, 106802 (2014).

[23] A. S. Rodin and A. H. Castro Neto, Phys. Rev. B 91, 075422 (2015).

[24] Y. Jiang, R. Roldán, F. Guinea, and T. Low, Phys. Rev. B 92, 085408 (2015).

[25] A. Favron, E. Gaufrès, F. Fossard, P. L. Lévesque, A. PhaneufL'Heureux, N. Y-W. Tang, A. Loiseau, R. Leonelli, S. Francoeur, and R. Martel, Nat. Mater. 14, 826 (2015).

[26] J. D. Wood, S. A. Wells, D. Jariwala, K.-S. Chen, E. Cho, V. K. Sangwan, X. Liu, L. J. Lauhon, T. J. Marks, and M. C. Hersam, Nano Lett. 14, 6964 (2014).

[27] J. O. Island, G. A. Steele, H. S. J. van der Zant, and A. Castellanos-Gomez, 2D Materials 2, 011002 (2015).

[28] S. Yuan, H. De Raedt, and M. I. Katsnelson, Phys. Rev. B 82, 115448 (2010).

[29] S. Yuan, R. Roldán, and M. I. Katsnelson, Phys. Rev. B 84, 035439 (2011).

[30] S. Yuan, T. O. Wehling, A. I. Lichtenstein, and M. I. Katsnelson, Phys. Rev. Lett. 109, 156601 (2012).

[31] A. H. C. Neto, F. Guinea, N. M. R. Peres, K. S. Novoselov, and A. K. Geim, Rev. Mod. Phys. 81, 109 (2009).

[32] S. Das Sarma, S. Adam, E. H. Hwang, and E. Rossi, Rev. Mod. Phys. 83, 407 (2011).

[33] M. I. Katsnelson, Graphene: Carbon in Two Dimensions (Cambridge University Press, Cambridge, 2012).

[34] S. Yuan, H. De Raedt, and M. I. Katsnelson, Phys. Rev. B 82, 235409 (2010).

[35] H. Qiu, T. Xu, Z. Wang, W. Ren, H. Nan, Z. Ni, Q. Chen, S. Yuan, F. Miao, F. Song et al., Nat. Commun. 4, 2642 (2013).

[36] S. Yuan, R. Roldán, M. I. Katsnelson, and F. Guinea, Phys. Rev. B 90, 041402 (2014).

[37] Y. Liu, F. Xu, Z. Zhang, E. S. Penev, and B. I. Yakobson, Nano Lett. 14, 6782 (2014).

[38] W. Hu and J. Yang, J. Phys. Chem. C 119, 20474 (2015).

[39] V. V. Kulish, O. I. Malyi, C. Persson, and P. Wu, Phys. Chem. Chem. Phys. 17, 992 (2015).
[40] R. Zhang, B. Li, and J. Yang, J. Phys. Chem. C 119, 2871 (2015).

[41] A. Ziletti, A. Carvalho, D. K. Campbell, D. F. Coker, and A. H. Castro Neto, Phys. Rev. Lett. 114, 046801 (2015).

[42] C. H. Lewenkopf, E. R. Mucciolo, and A. H. Castro Neto, Phys. Rev. B 77, 081410 (2008).

[43] S. Yuan, R. Roldán, H. De Raedt, and M. I. Katsnelson, Phys. Rev. B 84, 195418 (2011).

[44] E. H. Hwang, S. Adam, and S. Das Sarma, Phys. Rev. Lett. 98, 186806 (2007).

[45] Y. Zhang, V. W. Brar, C. Girit, A. Zettl, and M. F. Crommie, Nat. Phys. 5, 722 (2009).

[46] A. N. Rudenko, F. J. Keil, M. I. Katsnelson, and A. I. Lichtenstein, Phys. Rev. B 84, 085438 (2011).

[47] A. Principi, G. Vignale, M. Carrega, and M. Polini, Phys. Rev. B 88, 121405 (2013).

[48] M. Gibertini, A. Tomadin, M. Polini, A. Fasolino, and M. I. Katsnelson, Phys. Rev. B 81, 125437 (2010).

[49] M. Gibertini, A. Tomadin, F. Guinea, M. I. Katsnelson, and M. Polini, Phys. Rev. B 85, 201405 (2012).

[50] R. Kubo, J. Phys. Soc. Jpn. 12, 570 (1957).

[51] A. Hams and H. De Raedt, Phys. Rev. E 62, 4365 (2000).

[52] A. Politano and G. Chiarello, Nanoscale 6, 10927 (2014).

[53] N. D. Mermin, Phys. Rev. B 1, 2362 (1970).

[54] A. Agarwal and G. Vignale, Phys. Rev. B 91, 245407 (2015).

[55] G. F. Giuliani and G. Vignale, Quantum Theory of the Electron Liquid (Cambridge University Press, Cambridge, 2005).

[56] S. Das Sarma and E. H. Hwang, Phys. Rev. Lett. 81, 4216 (1998).

[57] O. V. Gamayun, Phys. Rev. B 84, 085112 (2011).

[58] R. Roldán and L. Brey, Phys. Rev. B 88, 115420 (2013).

[59] Q. Liu, X. Zhang, L. B. Abdalla, A. Fazzio, and A. Zunger, Nano Lett. 15, 1222 (2015).

[60] K. Dolui and S. Y. Quek, Sci. Rep. 5, 11699 (2015).

[61] J. M. Pereira and M. I. Katsnelson, Phys. Rev. B 92, 075437 (2015).

[62] J. Kim, S. S. Baik, S. H. Ryu, Y. Sohn, S. Park, B.-G. Park, J. Denlinger, Y. Yi, H. J. Choi, and K. S. Kim, Science 349, 723 (2015).

[63] A. Grigorenko, M. Polini, and K. Novoselov, Nature Photon. 6, 749 (2012).

[64] J. Schiefele, J. Pedrós, F. Sols, F. Calle, and F. Guinea, Phys. Rev. Lett. 111, 237405 (2013).

[65] T. Stauber, J. Phys. Condens. Matter 26, 123201 (2014).

[66] T. Low and P. Avouris, Acs Nano 8, 1086 (2014). 